\title{
A test method for analysing disturbed ethernet data streams
}

\author{
M. Kreitlow ${ }^{1}$, F. Sabath ${ }^{1}$, and H. Garbe ${ }^{2}$ \\ ${ }^{1}$ Bundeswehr Research Institute for Protective Technologies and NBC Protection, Munster, 29633 Germany \\ ${ }^{2}$ Institute of Electrical Engineering and Measurement Technology, Leibniz University Hannover, Hannover, 30167 Germany
}

Correspondence to: M. Kreitlow (matthiaskreitlow@bundeswehr.org)

Received: 13 January 2015 - Revised: 12 May 2015 - Accepted: 22 May 2015 - Published: 3 November 2015

\begin{abstract}
Ethernet connections, which are widely used in many computer networks, can suffer from electromagnetic interference. Typically, a degradation of the data transmission rate can be perceived as electromagnetic disturbances lead to corruption of data frames on the network media. In this paper a software-based measuring method is presented, which allows a direct assessment of the effects on the link layer. The results can directly be linked to the physical interaction without the influence of software related effects on higher protocol layers. This gives a simple tool for a quantitative analysis of the disturbance of an Ethernet connection based on time domain data. An example is shown, how the data can be used for further investigation of mechanisms and detection of intentional electromagnetic attacks.
\end{abstract}

\section{Introduction}

Ethernet following the IEEE 802.3 standard (IEEE Standard Association, 2012) is a technique, which is used in many network environments. At least the lowest level in big network installations is usually realised with CAT5e copper twisted pair lines for signal transmission. This enables for data transmission using the variant 1000BASE-T, which is also known as Gigabit Ethernet. Twisted pair lines of the category CAT5e do not require a metallic shielding. Therefore, these lines are more susceptible against radiated electromagnetic interferences. Especially intentional interferences have been subject of many investigations over the past years (Mojert et al., 2001; Jeffrey et al., 2004; Parfenov et al., 2008; Brauer, 2010). Amongst others, it was shown that interference from high-power electromagnetics (HPEM) can have a dramatic impact on data transmissions over Ethernet networks.

Typically the effects can be perceived on the application layer with regard to their criticality, e.g. when the data trans-

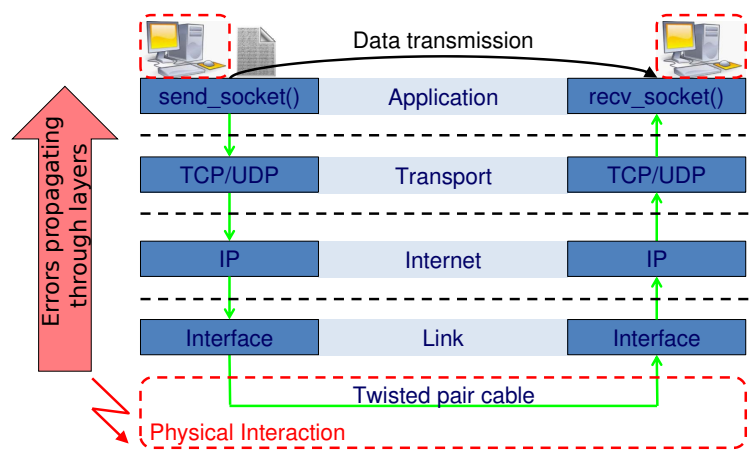

Figure 1. Communication with the TCP/IP reference model.

mission rate of a file transfer drops significantly or an interactive network application like voice-over-IP shows big lags (Sabath, 2008). In practise, it is very hard to find out what exactly is causing such a behaviour as the effects on application layer are normally not directly linked to the physical interaction. Figure 1 illustrates the process of logical communication within modern IP networks and physical interaction on the basis of the TCP/IP reference model (Postel, 1981a). This model can be considered as simpolified breakdown of the more general OSI layer model (ITU, 1994).

Errors within the communication can be caused by a hardware problem, a bottle-neck in the network infrastructure due to high utilization or even by a intentional electromagnetic interference (IEMI). Observing and detecting the lastmentioned is a real challenge. Reliable detection with field monitors is still subject of ongoing research and in big network infrastructures an effective field monitoring will probably be a high cost factor (Adami et al., 2014).

Therefore a new approach shall be discussed which allows for observation of disturbed data transmissions over an Ethernet network. Software-related effects on higher protocol 
layers, like control algorithms reacting to data errors, have to bypassed. It will be shown that a practical software implementation of a new test method gives a finely granulated time resolution of Ethernet frames and disturbances on the physical layer. This data can be used for further analysis to detect IEMI. Additionally this new method allows for testing network equipment with comparable results independently from user applications and specific software environments.

\section{Error mechanisms in classical TCP based communication}

Many network applications, such as file transfers, require a reliable data transmission. Altering or loss of data is unacceptable. Therefore typical techniques, like the file transfer protocol (FTP), utilize the transmission control protocol (TCP) on the transport layer, as this protocol offers integrated security measures. TCP operates on top of the IP protocol (Postel, 1981b). Whereas IP is only responsible for the logical routing of data packets from one destination to another through the network topology, TCP opens the communication endpoint for an application.

When testing a network for effects due to EMI, traffic has to be generated. This is often done by simply copying a file from one computer to another while the interference is present (Adami et al., 2012). This approach presents a problem, as the TCP protocol's integrated security mechanisms will react to occurring packet losses and delays. Therefore, the perceived effects are not linked to the physical processes on the network cables or within the computers. In van Leersum (2013) and Kreitlow et al. (2014) it was shown that an observed drop of the transmission speed can be a result of the control algorithms, although the physical degradation of the network is not very strong.

To conclude this, it can be said that investigating an IT network under the influence of EMI with TCP based applications will only give information on this specific network application in a specific network environment. This data is hardly comparable, as the TCP implementation will act as a "black box". To overcome these issues s new method shall be implemented.

\section{Design of a new test method}

The basic idea for a new measuring method is not to use a "black box" software like a FTP server/client to generate load on the network, which can be observed using network sniffing tools like Wireshark. Rather the data stream itself shall be generated in a well defined way without the influence of software based control algorithms. To achieve this, the user datagram protocol (UDP) will be used. UDP is also a part of the network stack of every modern operating system and operates on top of IP like TCP does (Postel, 1980).
Compared to this, UDP is a stateless protocol that provides no security and integrity checks on the transport layer. If a data packet gets lost or the data gets corrupted within an UDP connection, there will be no way to detect and handle this on protocol level as opposed to TCP. This makes UDP a very simple protocol with less overhead, but for this reason the application itself has to handle segmenting, flow-control and especially loss or corruption of data. Exactly this behaviour of UDP will be used to detect interferences without having influences of unknown control algorithms.

\subsection{Testing procedure}

As stated before the new method is based on UDP. It consists of a client, which requests a data stream from the server addressed by its IP address. The request itself is sent as an UDP datagram and defines all necessary testing parameters. In particular these are the test duration in seconds and the payload, which is the same for each packet to be sent. The payload size also defines the actual size of the packet. Additionally, there is a possibility to define delay-times for throttling the data transmission by inserting a delay after each UDP datagram. This is usually set to zero to achieve the maximum speed.

After sending the request to the server, the client immediately starts listening for incoming UDP datagrams originating from the servers IP address. The server will process the request and start sending UDP datagrams back to the clients IP address. The first four bytes of each datagram represent an 32-bit integer number, which acts as a sequence number. For each packet this number is increased consecutively, then the packet is filled up with the given payload up to the defined size. This process is running in a loop, that stops, when the server has been sending data for the specified duration.

On the other side of the connection the client will wait for incoming data. If a UDP datagram from the sever arrives, the client will check first check whether the payload specified in the request has been altered. This can be handled as a corruption. In practical tests this will actually never happen as data corruption due to bit flips will cause the frame check sequence of this Ethernet frame to fail. If this happens, the whole frame will be discarded. If an UDP datagram with the correct payload arrives, the sequence number will be read from the first 4 bytes of the datagram. This sequence number in combination with a time stamp is consecutively logged to a file. The client will stop its receiving and logging process, when it receives no datagrams for a specified time. After each test run, the logged data can be analysed off-line. This file will have the following format, where the first column is the time stamp in microseconds and the second column is the sequence number: [time stamp];[SEQ\#];.

The whole process is shown in Fig. 2 as a flow chart. The structure of each resulting Ethernet frame is illustrated in in Fig. 3. It is obvious that each Ethernet frame corresponds to exactly one UDP datagram and therefore to one unique sequence number. If an Ethernet frame is discarded or lost, 


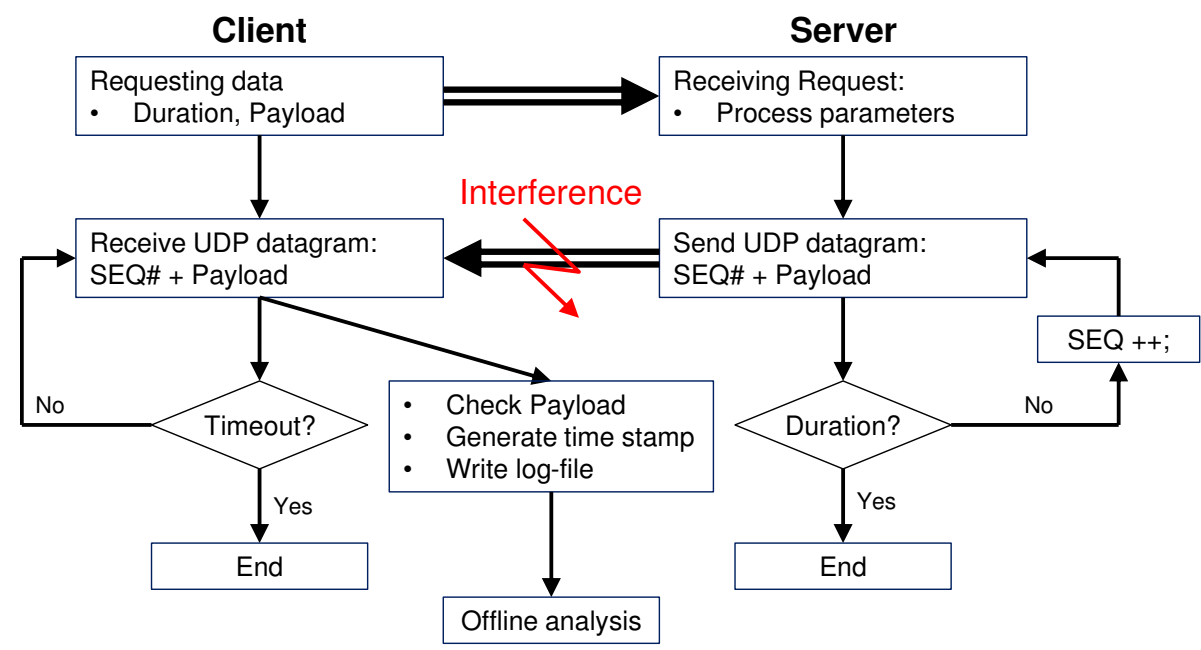

Figure 2. Flowchart for the test procedure.

\begin{tabular}{|c|c|c|c|c|c|c|}
\hline 8 bytes & 14 bytes & 20 bytes & 8 bytes & $18-1472$ bytes & 4 bytes & 12 bytes \\
\hline $\begin{array}{c}\text { Preamble }+ \text { start } \\
\text { of frame delimiter }\end{array}$ & Ethernet header & IP header & UDP header & SEQ\# + payload & $\begin{array}{c}\text { Frame check } \\
\text { sequence }\end{array}$ & \begin{tabular}{l} 
Interframe gap \\
\hline
\end{tabular}
\end{tabular}

Figure 3. Frame structure.

this can be directly seen in the log file as the sequence number in the corresponding UDP datagram will not occur, because UDP uses no techniques like retransmission or error correcting for securing the data transmission. Also all following Ethernet frames will not be affected by a single event due to the absence of flow control algorithms. By using this behaviour of UDP and the implementation above it is possible to detect interferences with the data transmission with a resolution down to single Ethernet frames.

\subsection{Implementation}

For a of concept the method was implemented in $\mathrm{C}$ as a Win32 application. The software has a user interface as shown in Fig. 4. An off-line analysis of the log file was done in MATLAB. However, there are some limitations in this implementation. As every UDP datagram is unique and must be generated in real-time, the maximum transmission speed is currently limited to $7.5 \mathrm{Mbyte}^{-1}$ at the moment. This is equivalent to approximately 5000 full-sized Ethernet frames ( 1538 bytes frame ${ }^{-1}$ ) per second. Using highresolution timers from Microsoft Windows, the time resolution is basically in the range of microseconds. Actually the absolute precision of this timers differs up to $10 \%$ from the exact time in this testing set-up. This is a consequence from the fact, that a high resolution and a good absolute accuracy with negligible drift, while summing up each small time step, is not possible at the same time using the software defined clock of an operating system.

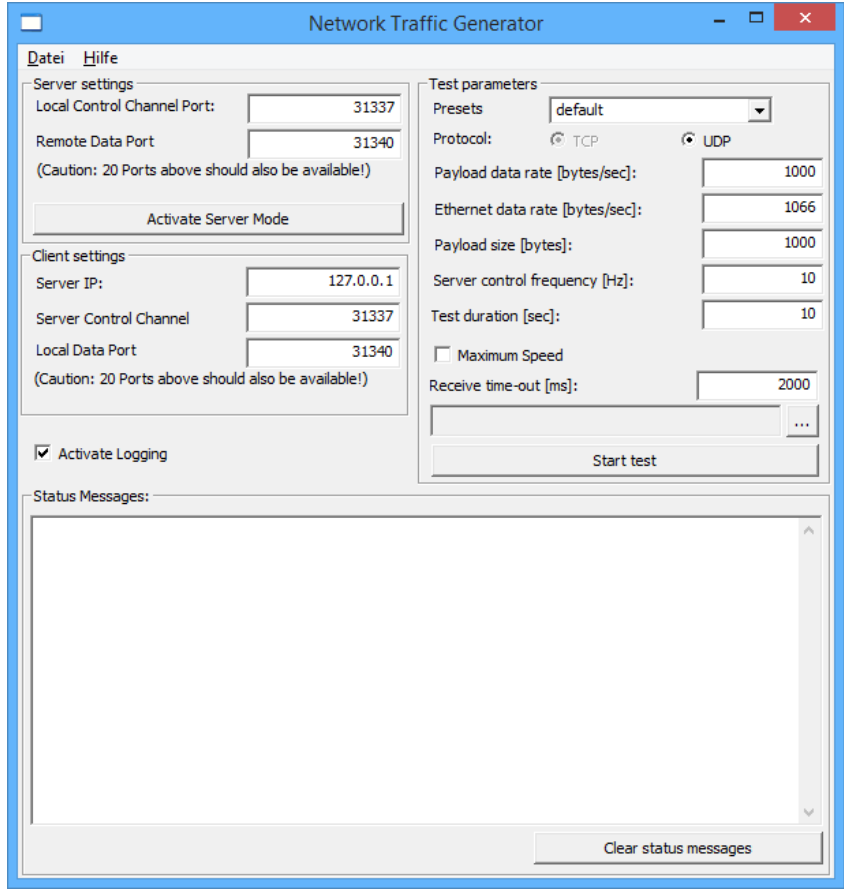

Figure 4. User interface of the test software.

\section{Test results}

The new method was used to perform some test of a IT network under the influence of IEMI. A simple topology as shown in Fig. 5 was set up. Two computers PC1 and PC2 are 


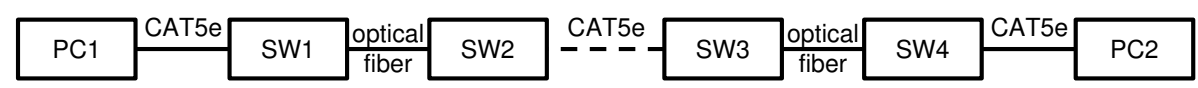

Figure 5. Topology of the test network.

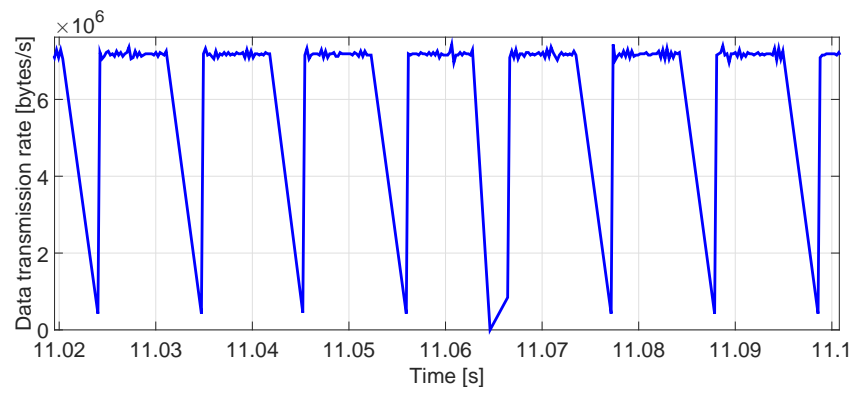

Figure 6. Data transmission rate over time.

connected over a line of switches SW1 to SW4. The computers are connected to the switches using CAT5e twisted pair cables. The switches SW2 and SW3 are also connected by twisted pair cables. SW1 and SW2 as well as SW3 and SW4 are linked with an optical fibre. Using an optical connection between these switches ensures that electromagnetic disturbances can not spread out over the whole network when only one component is exposed to interferences.

\subsection{Measuring data transmission rate}

Each line in the log file represents one UDP packet and therefore one Ethernet frame. The size of the frame is defined within in the test software. This information can be used to calculate the current data transmission rate $R$ on the lowest layer as

$R_{n}=\frac{S}{t_{n}-t_{n-1}}$,

where $S$ is the size of the complete Ethernet frame, $t_{n}$ is the time when the actual frame arrived and $t_{n-1}$ is the time when the previous frame arrived.

Figure 6 shows the result for an experiment where PC2 was exposed to ultra wide band (UWB) pulses with a a fieldstrength of $7.5 \mathrm{kV} \mathrm{m}^{-1}$ and a pulse repetition frequency of $100 \mathrm{~Hz}$, while PC2 was sending data to PC1. The data shows that the data transmission rate drops with every UWB pulse, but almost no data gets corrupted. In this detailed view only at $11.065 \mathrm{~s}$, where the data rate drops to zero, a packet loss actually happens. From this data it can be deduced, that the data transmission itself is not directly affected most of the time, but the PC delays sending new data for a short time, when it is affected by an UWB pulse.

This time domain data can be used to detect intentional attacks on computers as the pulse repetition frequency is reflected in the data transmission rate measured with the new method. Otherwise it would be hard to detect due to the ab-

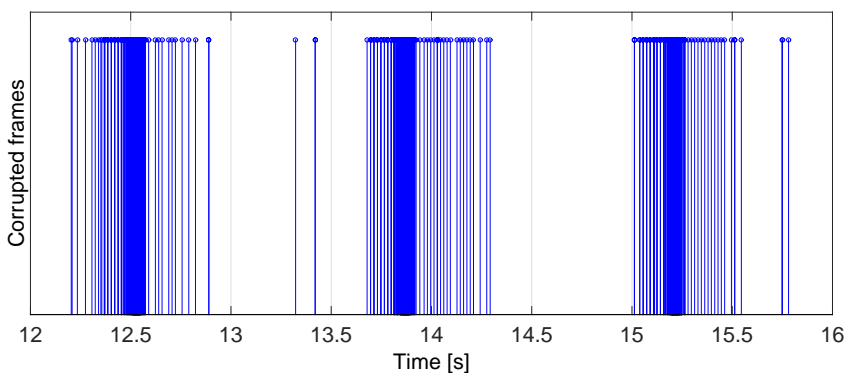

Figure 7. Corrupted Ehernet frames over time.

sence of exorbitant packet loss, although the transmission speed within applications will slow down.

\subsection{Measuring of packet losses}

Corrupted Ethernet frames can be detected by enumerating the sequence numbers in the log file. Without disturbances they should increase by one with each line. If the difference between two sequence numbers of two subsequent lines in the $\log$ file is higher than one, this indicates the number of missing Ethernet frames. The time stamps give the time window for the data corruption.

Another experiment was done, where an error current was directly coupled into the twisted pair cable between SW2 and SW3. The current also had the shape of an UWB pulse and the pulse repetition frequency was set to $20 \mathrm{kHz}$. Figure 7 shows at which time Ethernet frames get corrupted due to the interference. Each line in the diagram represents a corrupted frame. Although the disturbance source was permanently activated, the errors occur in bursts. The reason for this is a beat effect between the the packet rate $(\sim 5000 \mathrm{~Hz})$ and the repetition frequency of the source.

The data show that it is possible to catch single disturbance for a data stream with a high resolution in time. However due to the limited packet rate not every single event on the link layer can be caught as the software is not able to utilize the network line by $100 \%$. But if an effect occurs while the test software occupies the network line, it will be detected by this method. This makes the approach behind this method suitable especially for detecting repetitive interferences.

\section{Conclusions}

In this paper the challenges, when assessing the disturbance in a real world Ethernet set-ups, have been discussed. It has to be distinguished between the hardware effects and softwarerelated effects to get comparable results. To overcome this 
issue, a new test method was developed and implemented as a simple user application using the stateless user datagram protocol. In contrast to observing normal data transfers from e.g. FTP servers/clients, the effects on application layer perceived with this new method are directly linked to the disturbances on the physical layer. The results of the method are presented as time-domain data within a resolution in the range of approximately a few hundred microseconds. This data can be used for further investigations using known techniques of signal analysis to detect intentional interferences.

The implementation of the test-software is a proof of concept and still under development status. The source code can be requested from the corresponding author.

Edited by: F. Gronwald

Reviewed by: S. Dickmann and one anonymous referee

\section{References}

Adami, C., Braun, C., Clemens, P., Jöster, M., Suhrke, M., and Taenzer, H.-J.: High Power Microwave Tests of Media Converters, International Symposium on Electromagnetic Compatibility 2012 (EMC Europe), Rome, Italy, 17-21 September, 2012.

Adami, C., Braun, C., Clemens, P., Jöster, M., Suhrke, M., Schmidt, H.-U., and Tänzer, H.-J.: HPM-Detektionssystem mit Frequenzbestimmung, EMV Konferenz 2014, Düsseldorf, Germany, 11-13 March, 2014.

Brauer, F., ter Haseborg, J. L., Potthast, S.: Protection Circuits for IT Equipment under HPEM Conditions, AMEREM 2010, Ottawa, Canada, 2010.

IEEE Standard Association: IEEE 802.3-2012 - IEEE Standard for Ethernet, Section 3, 2012.

International Telecommunication Union (ITU): Telecommunication Standardization Section Sector of ITU (ITU-T), Information technology - Open Systems Interconnection - Basic Reference Model: The basic model, Recommendation X.200, 1994.

Jeffrey, I., Gilmore, C., Siemens, G., and LoVetri, J.: Hardware invariant protocol disruptive interference for 100BaseTX Ethernet communications, IEEE Transactions on Electromagnetic Compatibility, 46, 412-422, 2004.
Kreitlow, M., Garbe, H., and Sabath, F.: Influence of Software Effects on the Susceptibility of Ethernet Connections, IEEE International Symposium on Electromagnetic Compatibility EMC, Raleigh, NC, USA, 3-8 August, 2014.

Mojert, C., Nitsch, D., Friedhoff, H., Maack, J., Sabath, F., Camp, M., and Garbe, H.: UWB and EMP Susceptibility of Microprocessors and Networks, 14th International Zürich Symposium \& Technical Exhibition on Electromagnetic Compatibility, Zurich, Switzerland, 20-22 Februar, 2001.

Parfenov, Y. V., Kohlberg, I., Radasky, W. A., Titov, B. A., and Zdoukhov, L. N.: The Probabilistic Analysis of Immunity of a Data Transmission Channel to the Influence of Periodically Repeating Voltage Pulses, Asia-Pacific Symposium on Electromagnetic Compatibility \& 19th International Zurich Symposium on Electromagnetic Compatibility, Singapore, 283-286, 2008.

Postel, J.: User Datagram Protocol, RFC 768, USC/Information Sciences Institute, available at: http://tools.ietf.org/rfc/rfc768.txt, 1980.

Postel, J. (Ed.): Internet Protocol, DARPA Internet Program Protocol Specification, RFC: 791, Defense Advanced Research Projects Agency (DARPA), Arlington, Virginia, USA, available at: http://tools.ietf.org/rfc/rfc791.txt, 1981a.

Postel, J. (Ed.): Transmission Control Protocol, DARPA Internet Program - Protocol Specification, RFC: 793, Defense Advanced Research Projects Agency (DARPA), Arlington, Virginia, USA, available at: http://tools.ietf.org/rfc/rfc793.txt, 1981 b.

Sabath, F.: Classification of electromagnetic effects at system level, Proceedings of the 2008 International Symposium on Electromagnetic Compatibility (EMC Europe), Wroclaw, Poland, 18-22 August 2008, 1-5, 2008.

van Leersum, B. J. A. M., Buesink, F. J. K., Bergsma, J. G., and Leferink, F. B. J.: Ethernet susceptibility to electric fast transients, Proceedings of the 2013 International Symposium on Electromagnetic Compatibility (EMC Europe), Brugge, Belgium, 2-6 September 2013, 29-33, 2013. 